\title{
On Some Effects of Communication Barriers on the Quality of Candidate Performance and Examiner Judgements
}

\author{
Lilit Bekaryan \\ Russian-Armenian (Slavonic) State University \\ Irena Gyulazyan \\ American University of Armenia
}

\begin{abstract}
$\mathrm{C}$ ommunication is generally perceived as the result of complementary processes that operate at intrapersonal and interpersonal levels. At the interpersonal level, communication processes enable participants to produce and comprehend messages, whereas at the intrapersonal level, communication procedures help participants simultaneously affect and be affected by one another. The first type of interaction is particularly inherent in the speaking modules of such international exams, as IELTS, BEC, FCE or other Cambridge Main Suite Exams. All of the mentioned modules seek to assess the candidates' ability to speak on a variety of topics. The speaking modules always have the same format, which implies that the candidates are aware of the conventional event sequences that are expected of them, as well as know what their contribution to the test should be. At the same time, the candidates should be aware of the common language norms and maxims to be able to adequately respond to the examiners' speech acts to avoid barriers and misunderstanding. In this respect, the examinercandidate interaction can prove to be poor and ineffective if there are barriers to the communication process. We define every obstacle blocking the meaning of interaction in an examiner-candidate exchange as a communication barrier. The sources of barriers can vary from the cultural background of the individuals involved in communication to the first language interference, difficulties caused by the target language patterns, pronunciation, or accent.

The present paper identifies the types of communication barriers the interlocutors may experience in examination settings and considers to what extent these barriers might affect both the candidate's output and the assessment process.

To identify the nature and the effect of these barriers on the communication, we have studied a number of examiner-candidate interaction patterns, mostly retrieved from the speaking modules of IELTS exams. Considering the fact that the exams were administered in Armenia, most participants were either Armenians or Iranians.

It is interesting to note that though in these kinds of exchange, one of the interlocutors, namely the examiner, is the dominant speaker endowed with the so-called authority or power over his partner, both interlocutors tend to experience barriers in communication. Hence, if the examiner can face problems related to the components of assessment model because of the muddled messages of the examinee, wrong channels or inaccurate use of the target language by the examinee, for the candidate, the possible sources for language dissimilarity may reside in the first language interference, the insufficiency of
\end{abstract}


background knowledge, misunderstanding, culturally-sensitive topics discussed during the exam, etc. Let us consider an example:

\section{Examiner: Good afternoon!}

An Iranian Candidate: Thank you very much! (September, 2011)

As has already been mentioned, there are several stereotypic expressions that both the candidate and the examiner have to use within the exam script. To signal the candidate that the examination is about to start, the examiner makes use of the common system open signals, like Good morning or Good afternoon and in most cases where the candidate is from Iran receives the aforementioned response which seems irrelevant to communication. Although not everyone thanks you for being greeted, most Iranians do. The key to this strange behaviour can probably be sought in the fact that status is an important aspect of greeting etiquette in the Iranian culture. The lower status individual is to make the first gesture of the initial greeting.

With this in mind, some of the Iranian candidates especially these with a poor command of English wish to thank the examiners for making the first move to initiate the conversation.

The recent statistical data suggest that words account only for 40 percent of communication, whereas 60 percent of communication is non-verbal, implying an effective use of gestures, proxemics or eye contact. If the first two do not play such a significant role in the exam script, the second seems vital, as it only helps the candidate restore his confidence and assurance but also prompts the examiners if their message and instructions are compressible for the applicant. An examiner addressing an Iranian candidate can often regard him as indifferent, rude, or even not understanding when they answer the question without even looking at the interviewer. In Iran, though, a downward gaze is a sign of respect and even a defense measure for men, since staring at a woman is usually taken as a sign of interest, and can cause difficulties.

It is fair to acknowledge that the examiner's behaviour can also seem puzzling to the candidates. As already mentioned, the speaking modules are based on scripts with a sequence of wording that should be observed. For this reason, examiners are usually discouraged from providing such necessary system constraints as head nods or smiles, signaling that the message is getting through. Neither are they advised to practice their backchannelling skills through such fillers as 'yeah', 'right' or 'mmmhm' to avoid creating the impression of encouraging the candidate. Frequent are the cases, when the candidates puzzled by this obvious absence of feedback stare blankly at the examiner and ask him or her: 'Do you understand?' This may cause further barriers, because the examiner is not entitled to answer any questions like this.

Some barriers to communication can be overcome by consideration of the needs and understanding of the message recipients with slower speech or paraphrasing. Sometimes, when words fail to work, gestures come to help. For instance, with very low level candidates, examiners can mime or show with gestures what they mean for them to have more contribution to the overall discourse. For example: 
Examiner: Do you like painting?

Candidate: (confused) Penting? Explain, please.

Examiner (repeating the question and miming at the same time): Do you like painting?

Candidate: Ah, yes, I make pictures.

(July, 2011)

We can see that, despite some language inaccuracies in this exchange, there is no communication failure here due to the use of gestures. In the following exchange, though, the candidate is making an accurate but at the same time irrelevant contribution to the communication thread as she confuses birds with births.

Examiner: Are there birds where you live?

Candidate: As my country is a developing country and there no necessary

conditions, many families avoid having a lot of babies.

(September, 2011)

As already stated, the wording in the exam scripts reflects several functions, most of which tend to test the examinee's knowledge of English. However, in some cases the intent and the sentence meaning may not coincide, that is to say the syntactic form alone is not enough to tell the candidate how to interpret the speaker's intent. This is particularly applicable to directives when the candidate is requested to do something through polite imperatives like Can you, Could you or Would you ... ? Very often confusing such directives with questions, low level candidates tend to provide either yes or no answers, thinking they are being asked either a general or an alternative question. For example:

Examiner: Can I see your identification, please?

Candidate: Yes (not producing any required document).

or

Examiner: Can you tell me where you are from?

Candidate: No, Iran.

(June, 2011)

In the latter example the communicative goal of the exchange is achieved, as the candidate provides the answer, still the opening ' $n o$ ' of the utterance can seem a little distracting to the examiner.

Similar barriers can stem from insufficient knowledge of language structures, as in the following exchange, where a woman candidate has been asked to describe a dress she has worn to a special occasion.

Examiner: Do you have a photo of this dress?

Candidate (Puzzled) Now? No. (May, 2011) 
Most probably the woman thinks that she is being asked to produce the photo right away in the exam room.

The speaking component of IELTS consists of certain questions that dwell upon various topics. The examiners have to be very cautious in the choice of topics, as well, as what works well with the candidates.

Armenian examinees, for example, find the questions related to numbers very strange. When asked what numbers they have to remember, they usually stare at the examiner and request further explanation. Another question Do you like your name? apparently seems unsuitable for those candidates who have been named after their grandparents and bear long and old-fashioned names destined to oblivion.

In other cases, low-level candidates may misunderstand the message, as in the following exchange, which in its turn can impede the communication.

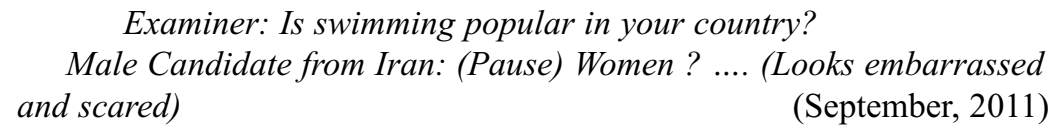

(September, 2011)

Obviously, representatives of different cultures have their own perception of strange or unreasonable questions. For instance, in Iran discussing women when conversing with men is highly unrecommended.

As we can see, the lack of shared assumptions and beliefs can lead to divergence in discourse strategies and communicative styles.

Thus, based on this small body of empirical research we can conclude that mishearing, discussing culturally sensitive topics, as well as making content-related wrong inferences can often trigger barriers in examiner-candidate communication.

While the barriers listed above do not always have a negative impact on communication, they appear to be risk factors in high-stake examination settings, where they might not only diminish the candidate's self-esteem, but also nurture misunderstanding, error and confusion, in this way inhibiting the assessment process.

\section{References:}

1. Countries and Their Cultures. Culture of Iran. $<\mathrm{http}: / /$ www.everyculture.com/GeIt/Iran.html>

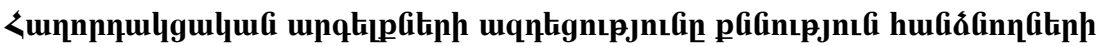

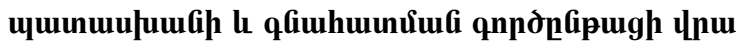

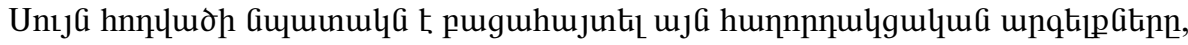

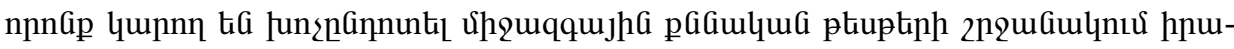




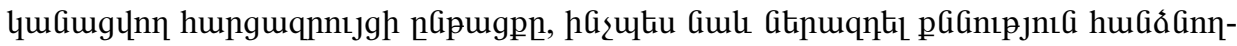

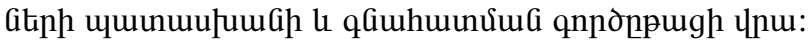

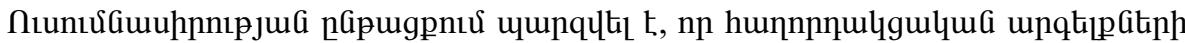

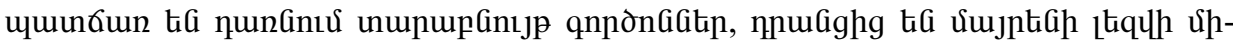

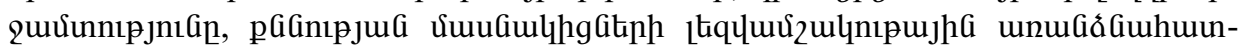

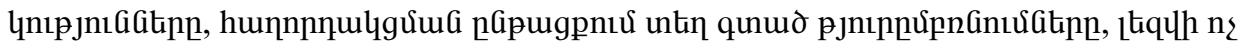

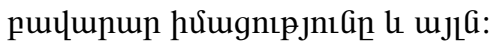

